TO EE PRESENTED AT THE 1993 PARTICLE ACCELERATOR CONFERENCE AND INTERNATIONAL CONFERENCE ON HIGH ENERGY ACCELERATORS

DALLAS, TEXAS MAY $1-5,1995$

CONF-9505/ $\dot{2}-155$ BNL-61234

\title{
COMBINED ELEMENT MAGNET PRODUCTION FOR THE RELATIVISTIC HEAVY ION COLLIDER (RHIC) AT BNL*
}

\author{
S. Mulhall, H. Foelsche, G. Ganetis, A. Greene, E. Kelly, S. Plate, E. Willen \\ Brookhaven National Laboratory, Upton, New York 11973-5000
}

The production of 432 combined element magnets for RHIC is well underway. These magnets consist of a superconducting corrector, a quadrupole, and a sextupole combined into an integrated cold mass which is inserted into a cryostat. Production experiences as well as test results are reported.

\section{INTRODUCTION}

A total of (432) $80-\mathrm{mm}$ aperture combined element magnets (CEM) are currently being fabricated at Brookhaven National Laboratory for installation into the RHIC ring. To date, (40) assemblies have been completed, with (60) additional units in various stages of assembly. These CEM's consist of various combinations of corrector, quadrupole, and sextupole elements, assembled together into a common cold mass containment and vacuum vessel. The majority of these magnets (294) are CQS assemblies, containing all three elements (see Fig. 1).

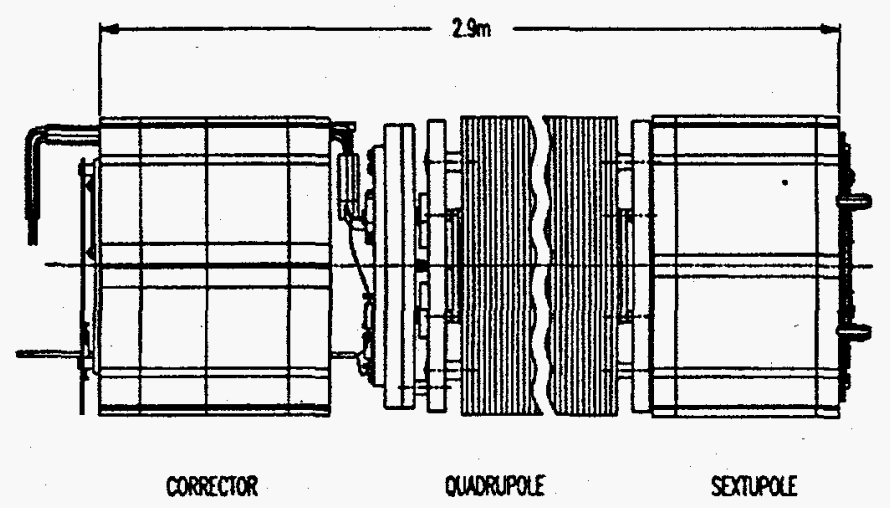

Fig. 1. CQS elements

The quadrupole and sextupole elements are manufactured industrially; the corrector elements are manufactured in-house at Brookhaven. The CEM program takes these three individual elements and assembles them, along with dozens of other components, into a single complete unit, ready for installation into the RHIC ring. Brookhaven elected to build the CEM in-house because of the large variety of types required, and the diverse origin of the many component parts.

Undertaking such a large-scale production program "inhouse" at Brookhaven has presented a unique opportunity for the laboratory to experience first-hand many of the production related

* Work supported by U.S. Department of Energy under Contract No. DE-AC02-76CH00016. issues and problems that are common to industry. In addition to technical issues that occur, these include problems of scheduling, manpower, documentation, parts deliveries, and Quality Assurance.

\section{CONFIGURATIONS}

The (432) CEM's consist of eighty separate and distinct "models", varying in required quantity from one to eighteen. In addition to the (294) standard CQS assemblies, there are seven other basic CEM types. These other assemblies do not contain the sextupole element and vary in design by incorporating a trimquadrupole in its place and/or the use of a different length quadrupole element. These CEM's are designated CQT4, CQT5, CQT6, CQ7, CQ8, CQ9, and CQ9 (Special). The total number of different configurations is brought to eighty by the incorporation of the following additional variables:

Corrector Type (five options)
Clockwise/Counterclockwise Ring Orientation
Quench Protection Diode Polarity (Quadrupole)
Beam Position Monitor Direction (Horiz/Vert/Both)
Presence of Cryogenic Recooler

\section{PRODUCTION}

\section{A. Plan}

The overall plan for producing CEM assemblies at Brookhaven, and the required tooling, had been developed during the RHIC magnet R\&D program over several years. In late 1993, following a pre-production program that consisted of two complete assemblies (using non-production tooling), the present CEM production schedule was developed. An aggressive schedule was adopted, in which production would run for roughly twenty-six months (ending October, 1996). Aftei an initial rampup to full production, a magnet delivery rate of one-per-day was targeted as an achievable goal. The sequence of producing the various models was arranged based upon providing the magnets required for the RHIC sextant test by early 1996 while minimizing perturbations to the production line due to tooling changeovers.

Detailed manpower plans were developed and coordinated with other Brookhaven magnet construction programs. A full-scale Quality Assurance program was implemented, encompassing virtually every aspect of the upcoming production: parts procurement, material control, production travelers, discrepancy reporting system, etc.... Magnet assembly drawings and procedures were developed (and continue in development as production of different models begins). 
Production readiness reviews were held in mid-1994 with Brookhaven scientists and engineers. Two independent production reviews were conducted including experts from private industry, and their recommendations incorporated into the program. Production of the first magnet began in June, 1994.

\section{B. Process}

The CEM is designed to incorporate many subsystems required in the machine into a single integrated unit that can be assembled and tested in a factory setting. Tooling is set up to provide accurate alignment between components, to minimize manual labor, and to reduce assembly errors. CEM assemblies are built to fit into specific positions in the RHIC lattice.

The CEM production process begins with the initial assembly of the individual elements onto a common fixture and the wiring of the center element (quadrupole) (Fig. 1). A fully automated dual-head MIG machine is used to weld the stainless steel shells around the elements. Subsequent assembly stations align and weld the end plates and support cradles. Electromechanical assembly comes next, including installation of the quench protection diode for the quadrupole. The beam tubebeam position monitor assembly is then installed, and the cold mass end volumes welded in place (Fig. 2).

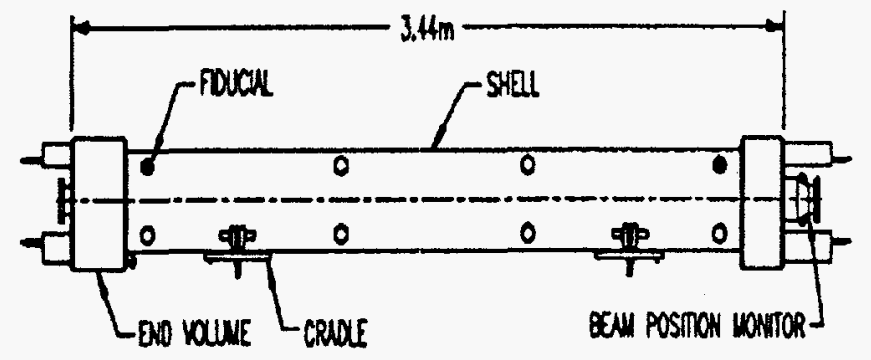

\section{CORRECTOR ENO}

SOTUPAE ENO

Fig. 2. CQS cold mass assembly

The next critical step is measurement of the alignment between the individual cold mass elements, followed by fiducial installation. This operation is performed on a granite surface plate using digital-mechanical instruments reading directly into a computerized data-base. The reliability of the measurements taken during this process has improved significantly since the start of the program, and will likely continue to evolve as data accumulates and opportunities for improvement occur. Following these alignment measurements, the completed cold mass is pressurized internally to $345 \mathrm{psig}$, while simultaneously drawing a vacuum on the outside. Leak tightness down to $2 \times 10^{-10} \mathrm{Std}-\mathrm{cc} \mathrm{He} / \mathrm{sec}$ is thus assured.

Insulation blankets, pipes, heat shield, and support posts are then assembled to the cold mass and the entire sub-assembly is inserted into its vacuum vessel (Fig. 3). Final electrical connections and polarity checking are then completed. The finished assembly is vacuum leak tested and, following warm magnetic measurements, ready for delivery to either horizontal cold testing or directly to the RHIC ring.

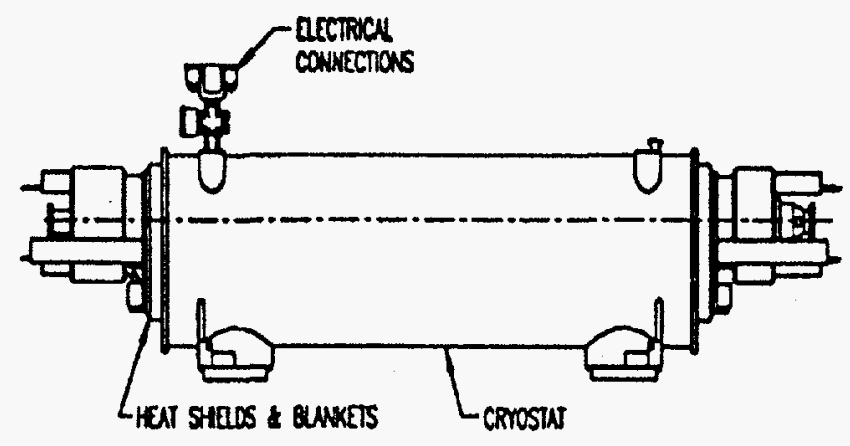

CORRCCIOR OO

SDTupar 20

Fig. 3. Completed CQS assembly

\section{STATUS \& EXPERIENCE}

The CEM program is close to schedule with regard to magnets under construction, but has fallen behind in terms of completed units. This shortfall is primarily due to parts delivery problems. These delivery problems have only recently been resolved, and an aggressive effort is being made to clear the backlog of partially assembled magnets.

To date, some twenty-three CQS magnets have been successfully cold tested in the horizontal test facility. Corrector and sextupole elements have been ramped to the limit of their leads ( $20 \%$ above their maximum operating current), with only one quench among all the magnets. Quadrupole elements consistently quench at $7.5 \mathrm{kA}$ with little training, $50 \%$ above their nominal operating current. Note that CQS quadrupole quench currents are below those obtained for the individually tested quadrupole elements because of the heat load from the warm finger inserted into the CQS for magnetic measurements [1].

In bringing the CEM production program to this point, the following experiences, in the nature of "lessons learned", are noteworthy:

\section{A. Tooling}

he ability to build pre-production magnets using the final production tooling would have resulted in a significantly smoother start-up in the actual construction program. Tooling shake-outs and modifications contributed to delays in program start-up.

\section{B. Parts}

The issue of parts availability has proven to be more difficult than anticipated. Some of these issues are attributable to allowing insufficient lead time to the vendors, but most are not. Problems related to the major contracts run the gamut from vendors simply falling behind schedule to their outright inability to produce parts to specification. These problems represent the primary reason for the program having fallen behind schedule. It is unclear whether these problems could have been mitigated by more diligent up-front efforts (e.g. using Requests for Proposals and technical evaluation boards). Given current government 


\section{DISCLAIMER}

Portions of this document may be illegible in electronic image products. Images are produced from the best available original document. 
contracting procedures and the fact that private industry is similarly plagued by problems of this nature, it may well be that these situations should be classified as "essentially unavoidable". In any event, it is apparent that Brookhaven staff was too optimistic in this regard, and in the future should be more cautious.

\section{Technical lssues}

Relatively few technical issues have arisen during the initial production phase, and these might be classified as "nagging" rather than "critical" in nature.

Perhaps the primary technical issue to-date has been with regard to magnet alignment and distortions caused by welding. One such distortion was noted early-on: the welding of the cold mass support cradles to the shell caused an unacceptable bend in the entire cold mass assembly. It was then necessary to modify the welding procedures so that a roughly equal quantity of weld metal would be added directly opposite to the cradle welding, thus effectively canceling any distortion. Other distortions are caused by the welding of the fiducial dises, and by the welding of the cold mass end volumes.

To help quantify and deal with the welding distortions, it was found necessary to upgrade the alignment measurement data acquisition method. Fiducial measurements, for example, were initially taken mechanically, and recorded by hand; optical surveying was then performed as a check of accuracy. Since performing an optical survey on each cold mass is extremely time consuming, it was decided to upgrade the mechanical system by incorporating digital gauges and a computerized data collection system, generating on-line plots of magnet alignment. Initial results from the new system are very encouraging. It should be noted that the magneto-optical colloidal cell is being utilized on all CEM units as a final check of alignment data [2].

Another technical issue which arose early-on concerned the method of pressure/leak checking the cold mass assembly. A combined test is done, wherein the magnet, suspended in a test dewar, is pressurized intemally to 345 psig using helium while simultaneously drawing a vacuum on the outside of the assembly. In this condition, a leak detector monitors the dewar for helium leakage for 15 minutes. A successful test certifies the cold mass assembly to a maximum leak rate of $2 \times 10^{-10} \mathrm{Std} \mathrm{cc} \mathrm{He} / \mathrm{sec}$. In several instances, leaks have been detected in the cold mass (in the end volumes) that were not previously discovered when the individual component was given only a simple leak check. Under internal pressure, small movements and flexing occurs that reveal leaks that would otherwise go undetected. It is thus concluded that the combination pressure/leak test, replicating the actual in-service condition, is an extremely valuable production step.

\section{SUMMARY}

Ten months into actual magnet production, the construction of CEM assemblies has reached full-speed, with ongoing deliveries to the RHIC ring. Tooling shake-out is complete'and assembly technicians are well trained in their tasks. Some vendor problems remain and are being dealt with appropriately. A way has been found to deal with the technical issue of distortions caused by welding. Though start-up took longer than anticipated, the program appears poised for a successful run, with completion on or near schedule.

\section{ACKNOWLEDGEMENTS}

We acknowledge with pleasure the hard work and technical expertise of our industrial partners Northrop Gumman Corporation and Everson Electric.

\section{REFERENCES}

[1] J. Wei et al., "Field Quality Evaluation of the Superconducting Magnets for the Relativistic Heavy Ion Collider," Proc. PAC ' 95.

[2] D. Trbojevic et al., "Alignment and Survey of the Elements in RHIC," Proc. PAC '95.

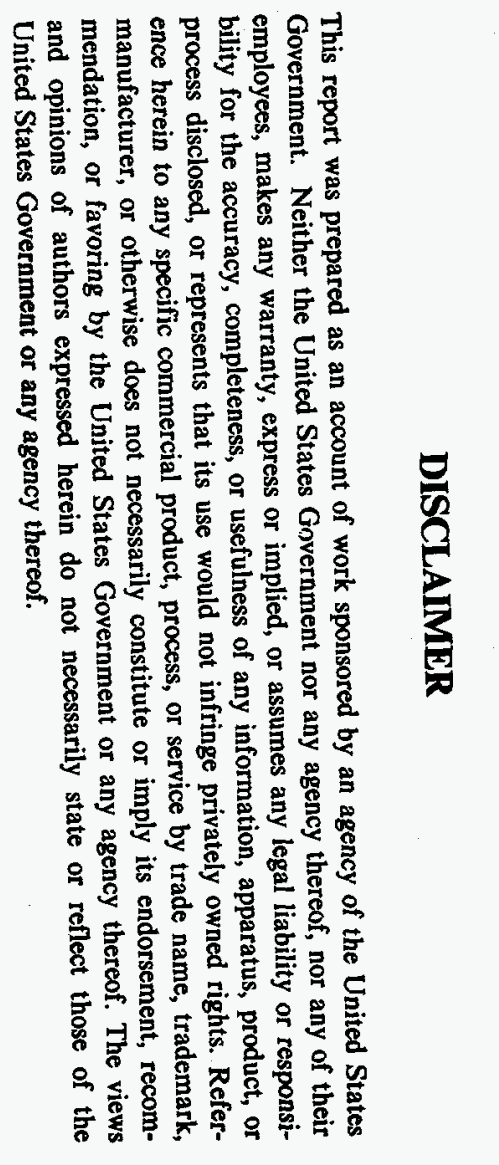

